\title{
Large-scale screening of hypothetical metal-organic frameworks
}

\author{
Christopher E. Wilmer', Michael Leaf', Chang Yeon Lee², Omar K. Farha², Brad G. Hauser², \\ Joseph T. Hupp ${ }^{2}$ and Randall Q. Snurrћ
}

\begin{abstract}
Metal-organic frameworks (MOFs) are porous materials constructed from modular molecular building blocks, typically metal clusters and organic linkers. These can, in principle, be assembled to form an almost unlimited number of MOFs, yet materials reported to date represent only a tiny fraction of the possible combinations. Here, we demonstrate a computational approach to generate all conceivable MOFs from a given chemical library of building blocks (based on the structures of known MOFs) and rapidly screen them to find the best candidates for a specific application. From a library of 102 building blocks we generated 137,953 hypothetical MOFs and for each one calculated the pore-size distribution, surface area and methane-storage capacity. We identified over 300 MOFs with a predicted methane-storage capacity better than that of any known material, and this approach also revealed structure-property relationships. Methylfunctionalized MOFs were frequently top performers, so we selected one such promising MOF and experimentally confirmed its predicted capacity.
\end{abstract}

$H^{2}$ ghly porous materials have widespread applications in the manipulation of small molecules for gas storage $e^{1-6}$, separating mixtures ${ }^{7-12}$, catalysis ${ }^{13,14}$, analysis and detection ${ }^{15-17}$. Among the many types of porous materials ${ }^{18,19}$, metal-organic frameworks ${ }^{19-21}$ (MOFs) have attracted particular interest because of their high surface area, porosity and stability ${ }^{22-25}$, as well as the ease with which they can be synthesized based on designs conceived a priori. This advantage stems from the use of modular molecular building blocks that self-assemble into predictable crystal structures ${ }^{26,27}$. As a result of this predictability and the abundance of known modular building blocks ${ }^{28-32}$, thousands of reports of novel MOFs have appeared over the past few years ${ }^{28}$, along with many in-depth investigations of their properties and functionality ${ }^{33}$. Although these numerous reports showcase the success of the modular building block approach, they also belie the underlying combinatorial difficulty of finding the MOF with the best material properties for a given application. Rather than mere thousands, there are millions of possible MOFs, even when libraries of fewer than 100 building blocks are considered (see Supplementary Information).

We leveraged the predictable assembly of building blocks into MOFs by systematically generating every possible structure within constraints (for example, no more than four unique building blocks per MOF) given an input library of building blocks, and for each structure we used rapid computational simulations to predict the material properties. Thus, for each hypothetical MOF we obtained a range of material properties (surface area, pore volume, pore-size distribution, powder X-ray diffraction pattern and methane-adsorption capability), and in doing so revealed unidentified structure-property relationships ${ }^{34}$ that could be recognized only from a global view of MOF structures.

Our work shares much in spirit with the database of hypothetical zeolites created by Earl and Deem ${ }^{35}$, recently screened rapidly by Haldoupis et al. ${ }^{36}$ for gas-adsorption properties. However, synthesis of novel zeolites is significantly more difficult than that of novel MOFs; less than 200 different zeolite structures have been synthesized to date ${ }^{37}$, compared to thousands of MOFs over a much shorter time period ${ }^{28}$. The number of MOFs is so large that there is considerable merit in also developing methods to screen rapidly the known experimental structures for desired properties, as has been done by Haldoupis et al. to identify candidates for kinetic separations ${ }^{38}$.

In addition to identifying structure-property relationships, we used this systematic approach to identify the best MOFs, from the vast sea of possibilities, for a representative, specific application: high-pressure methane storage. Based on structure-property insights from the database, we identified and synthesized the NOTT-107 MOF (ref. 39), which we predicted to have better methane-storage capacity at 35 bar than that of PCN-14 (ref. 40), the current world-record holding material for methane-storage capacity (computer simulations of the former showed higher methane storage than simulations of the latter). The experimental adsorption isotherm for methane in NOTT-107 agrees well with our predictions, which demonstrates the accuracy and utility of the systematic approach we describe.

\section{Results}

Generation procedure. The generation procedure creates hypothetical MOFs by recombining building blocks derived from crystallographic data of already synthesized MOFs (see Fig. 1a). Atoms are grouped into building blocks based on the reagents used in the actual synthesis. Any building block can combine with any other building block provided that the geometry and chemical composition local to the point of connection (marked by a purple $\mathrm{X}$ in Fig. 1b) is the same as that in crystallographically determined structures. Building blocks are combined stepwise, and when an atomic overlap occurs at a particular step a different building block or a different connection site is chosen, until all the possibilities are exhausted. Although the total number of steps in each generation process can vary, there are always three steps when, instead of adding a building block, a periodic boundary is imposed by connecting two building blocks (steps ii and iv in

'Department of Chemical and Biological Engineering, Northwestern University, 2145 Sheridan Road, Evanston, Illinois 60208, USA, ${ }^{2}$ Department of Chemistry, Northwestern University, 2145 Sheridan Road, Evanston, Illinois 60208, USA. *e-mail: snurr@northwestern.edu 

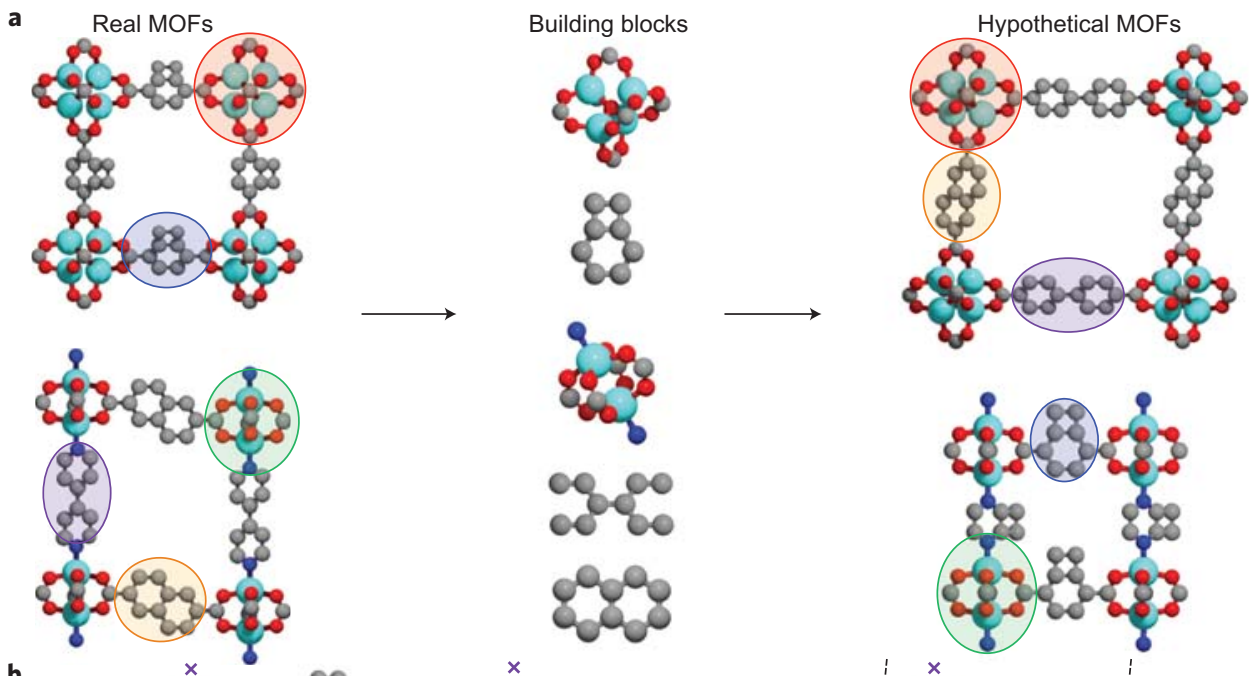

b

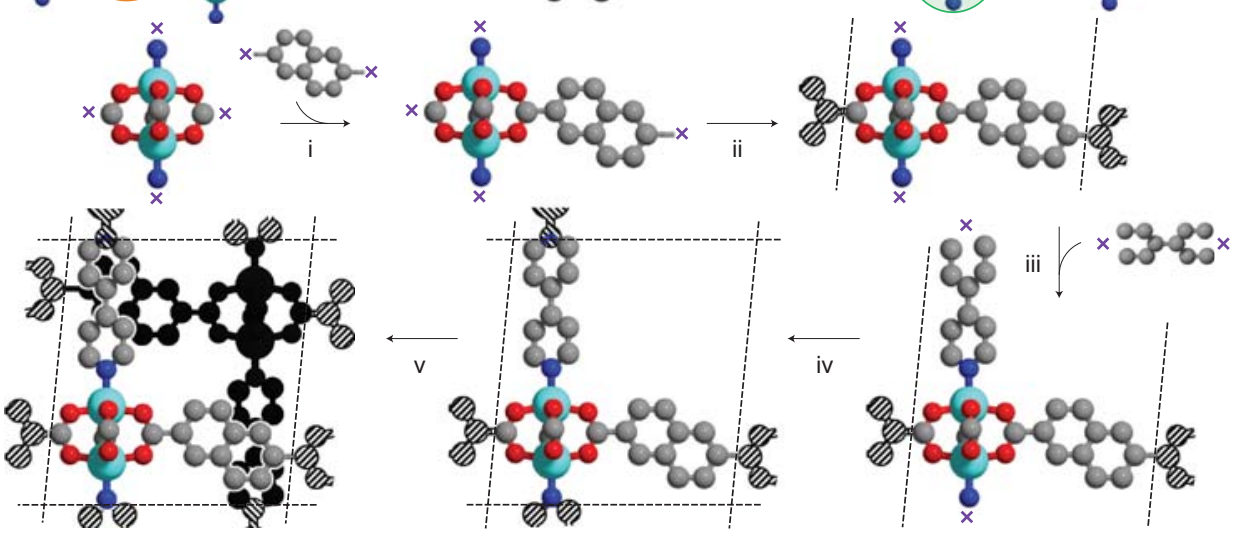

Figure 1 | Visual summary of the hypothetical MOF-generation strategy. $\mathbf{a}$, Crystal structures of existing MOFs were obtained from X-ray diffraction data (a, left) and subsequently divided into building blocks (a, middle) that then could be recombined to form new, hypothetical MOFs (a, right). $\mathbf{b}$, The recombination process occurs by stepwise addition of building blocks (i), which are attached at their connection sites (purple Xs). Building blocks are also connected across periodic boundaries (ii, hashed circles indicate mirror images). The process repeats (iii to iv) until all connection sites are utilized. An interpenetrated MOF may be generated if enough space exists ( $v$, black circles indicate atoms belonging to one of two interpenetrated frameworks). Grey, red, blue and turquoise spheres represent carbon, oxygen, nitrogen and zinc atoms, respectively. Hydrogen atoms are omitted for clarity.

Fig. 1b). When no more building blocks can be added, the crystal generation procedure is complete (for more details, see the Supplementary Information). No force field (or quantum mechanical) energy minimizations are involved; the pieces are connected according to the geometric rules that govern how the building blocks are connected in existing MOFs. The approach is very much like snapping Tinkertoys or Lego bricks together.

Structure validation. Broadly, there are three primary concerns when generating hypothetical crystal structures. Are the structures in an energetic minimum? Do generated hypothetical structures agree with experimentally measured structures? How sensitive are predicted physical properties to structural inaccuracies? To address these concerns, we compared a set of generated structures with their energetically relaxed counterparts by force-field minimizations and also with their experimentally measured structures. We considered the influence of these structural differences on predicted properties by investigating a specific application, in this case methane adsorption.

By choosing the appropriate building blocks, we generated crystal structures that resembled the MOFs HKUST-1 (ref. 41), IRMOF-1 (ref. 20), PCN-14 (ref. 40) and MIL-47 (ref. 42). These MOFs differ significantly in their pore topology and chemical composition. We refer to the generated structures as pseudo-HKUST-1, pseudo-IRMOF-1, pseudo-PCN-14 and pseudo-MIL-47 to indicate that, albeit not hypothetical, they are nonetheless not identical to empirical structures. As a test of the generation procedure, we allowed these pseudo-MOFs to relax their structures energetically by the Universal Force Field ${ }^{43}$ implemented in the Forcite module in Materials Studio ${ }^{44}$. For both the relaxed and nonrelaxed versions, superimposing the experimental and generated structures shows that the atoms match very closely, with every atom in the pseudo-MOFs shifted from its measured position typically by an average of less than $\sim 0.1 \AA$ (see Supplementary Information for the details).

Ultimately, systematic screening of hypothetical MOFs depends directly on the accuracy of the predicted properties rather than on the accuracy of the crystal structures. Hence, for each MOF structure (experimental, pseudo and pseudo after relaxation/optimization) we predicted computationally the methane adsorption isotherms at $298 \mathrm{~K}$ using grand canonical Monte Carlo (GCMC) simulations, as

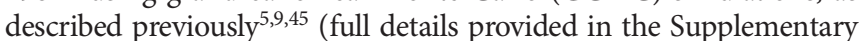
Information). The three methane-adsorption isotherms predicted computationally from the experimental, pseudo and pseudo after relaxation/optimization MOFs closely matched each other, in particular those of the experimental structures and the pseudo-MOFs. (No comparison is made in Fig. 2 with any isotherms obtained experimentally.) Düren and Snurr9 9 Düren et al. ${ }^{5,45}$ and Supplementary Fig. S9 show good agreement between simulation and experiment using the same GCMC methods. 

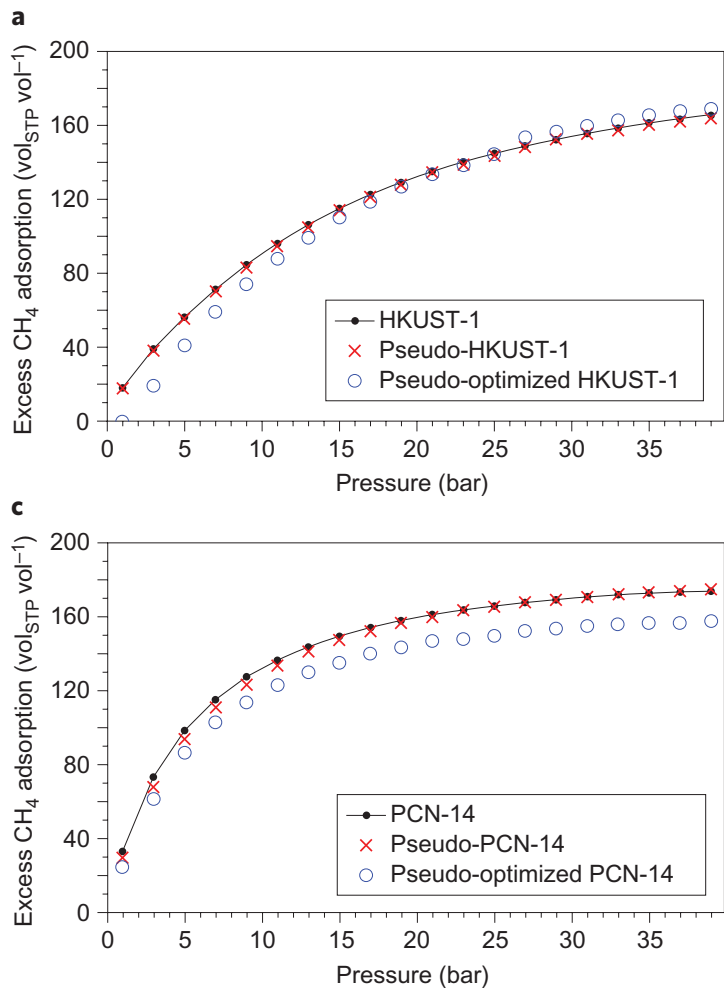

b
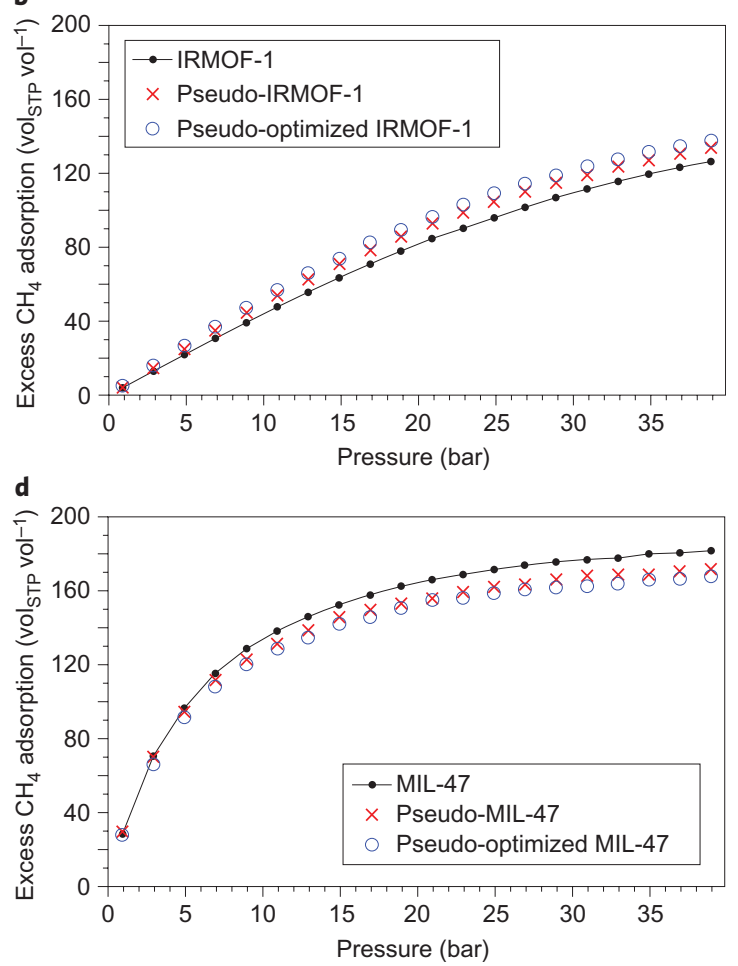

Figure 2 | Influence of structural variations on predicted methane adsorption. a-d, Comparisons of simulated methane adsorption isotherms at $298 \mathrm{~K}$ for the experimental (black lines), pseudo (red crosses) and pseudo-optimized (empty circles) structures of HKUST-1 (a), IRMOF-1 (b), PCN-14 (c) and MIL-47 (d) show good agreement.

Systematic screening. Having validated our capability to generate MOFs, we performed a systematic screening on a very large scale to identify promising MOFs for high-pressure, room-temperature methane storage. Vehicles that can be powered by natural gas, which is mostly methane, emit less greenhouse gas per mile travelled than gasoline vehicles, but it is a challenge to store sufficient quantities to cover typical driving distances ${ }^{46}$. Materials that meet the US Department of Energy target of $180 \mathrm{~cm}^{3}$ of stored methane at standard temperature and pressure $\left(\mathrm{cm}^{3}{ }_{\text {STP }}\right)$ per cubic centimetre at 35 bar and $298 \mathrm{~K}\left(\mathrm{~cm}_{\text {STP }}^{3} \mathrm{~cm}^{-3}\right.$, more generally $\mathrm{vol}_{\mathrm{STP}} \mathrm{vol}^{-1}$ ) could potentially lead to cost-effective highdensity storage tanks for vehicles powered by natural gas. In particular, recently it was demonstrated that MOFs have promise in this area ${ }^{5,40,47,48}$, but a material suitable for mass production is yet to be discovered.

For the generation procedure we chose 102 building blocks that varied significantly in their geometries, number of connection sites and chemical composition (see Fig. 3 for a partial list and Supplementary Information for the complete list). The building blocks fall conceptually into three groups: inorganic, organic and functional groups. Although the generation algorithm is normally blind to these distinctions, it was constrained in this case to produce crystals with at most one kind of inorganic building block, two kinds of organic building blocks and one kind of functional group. This constraint resulted in MOFs that were reasonable synthetic targets, with similar complexity to those reported commonly in the literature. This constraint could be lifted easily to investigate, for example, the 'multivariate' MOFs reported by Deng et al. ${ }^{49}$ that contain up to nine unique building blocks within one crystal.

By attempting every combination of building blocks, subject to the above constraints, 137,953 hypothetical MOF structures were generated in total. We screened each MOF for methane storage at 35 bar and $298 \mathrm{~K}$, but also calculated other properties such as surface area, void fraction, pore-size distribution and powder $\mathrm{X}$-ray diffraction pattern (structures and properties are accessible online at hmofs.northwestern.edu). Screening occurred in three stages: every MOF was subject to short 500-cycle GCMC simulations, then the top 7,000 MOFs were subjected to 2,500 cycle simulations and finally the top 350 from the second stage were subjected to 12,500 cycle simulations (Fig. 4; a cycle consists of $N$ Monte Carlo steps, where $N$ is the number of methane molecules present at any given point). The third stage (highest quality) GCMC predictions indicate that $\sim 300$ MOFs have higher methane-storage capacity at 35 bar than the current world record $\left(230 \mathrm{vol}_{\text {STP }} \mathrm{vol}^{-1}\right)$. For example, the best hypothetical MOF (shown in Fig. 4f) is predicted to store $\sim 267 \mathrm{vol}_{\text {STP }} \mathrm{vol}^{-1}$ methane at $298 \mathrm{~K}$.

In addition to identifying promising candidates for methane storage, we can also learn from trends shown by this type of 'global view' of possible MOFs. For example, a clear linear relationship exists between volumetric methane adsorption and volumetric surface area (Fig. 5a), but not between volumetric methane adsorption and gravimetric surface area (Fig. 5b). Maximizing gravimetric surface area is a common strategy in MOF design, but going past the optimal point $\left(\sim 2,500-3,000 \mathrm{~m}^{2} \mathrm{~g}^{-1}\right)$ only worsens the methane-storage capability. Despite diverse topological and chemical differences, all of the best hypothetical MOFs share a remarkably narrow cusp of optimal void fractions around $\sim 0.8$ (Fig. 5 c). Similar to gravimetric surface area, a pore volume that is larger than optimal has a steeply negative effect on methane storage. MOFs with methyl, ethyl and propyl functional groups dominate the best performers; over 75\% of MOFs with adsorption over $205 \mathrm{vol}_{\text {STP }}$ vol $^{-1}$ contain methyl, ethyl or propyl functional groups, as shown in Fig. 5d,e. Previous isolated reports found that the optimal pore size for methane storage is either large enough for a single methane molecule $^{50}$ or large enough for two ${ }^{5}$. In fact, the answer is both: the 


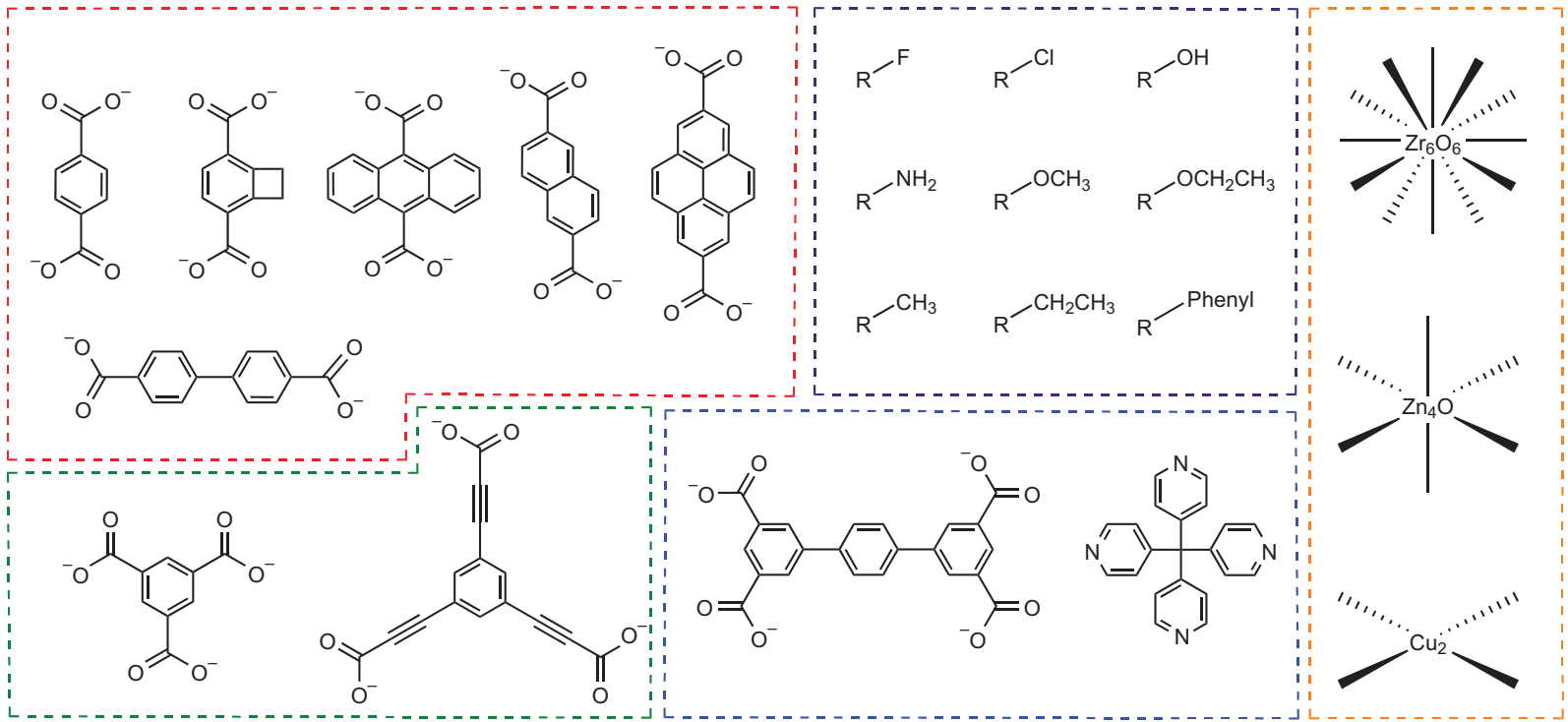

Figure 3 | Partial list of building blocks used in the large-scale screening process. The purple, red, green and blue groupings show examples of one-, two-, three- and four-connected building blocks, respectively. The one-connected building blocks are functional groups and the others (that do not contain metals) are organic building blocks. The orange grouping shows three inorganic buildings blocks that are four-, six- and 12-connected. (See Supplementary Information for the complete list.)
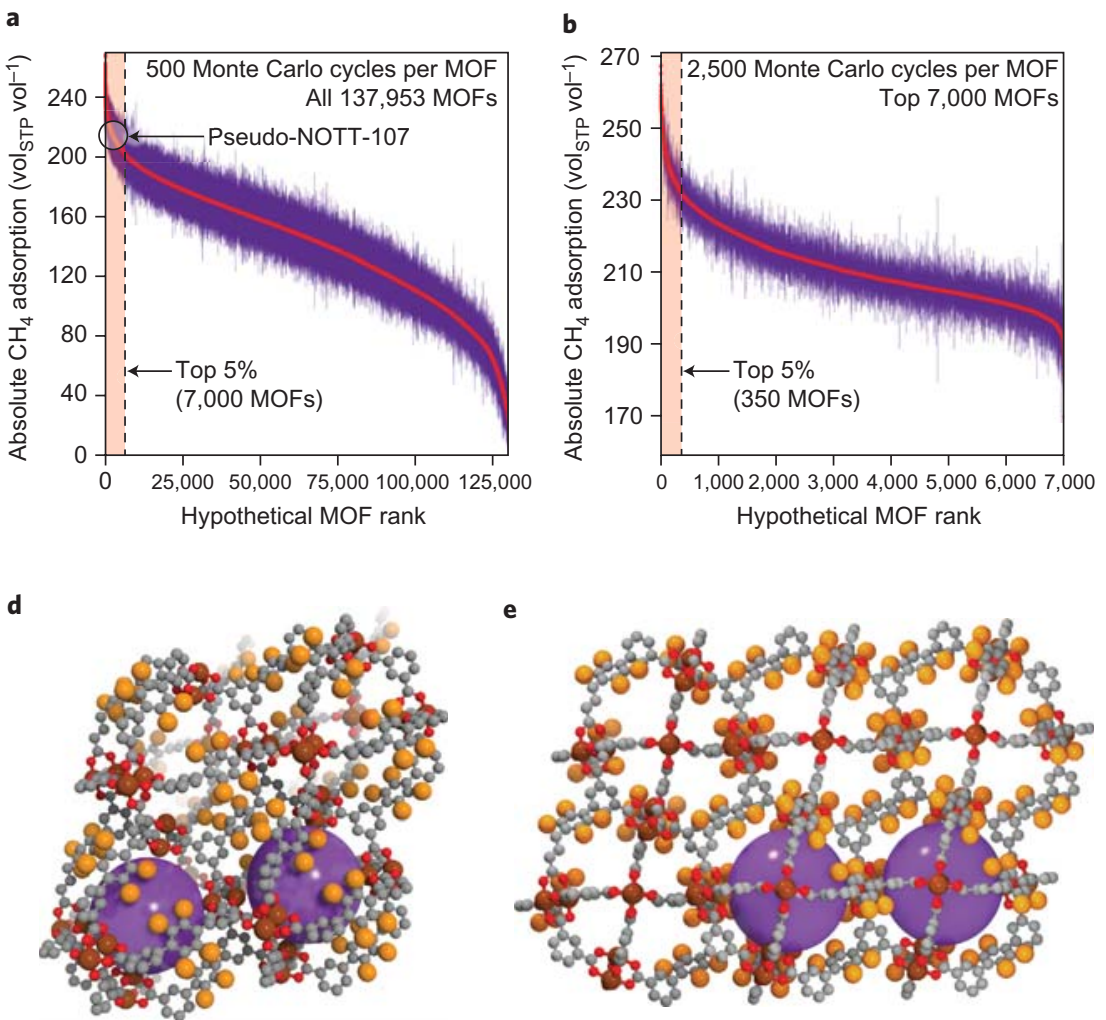

e

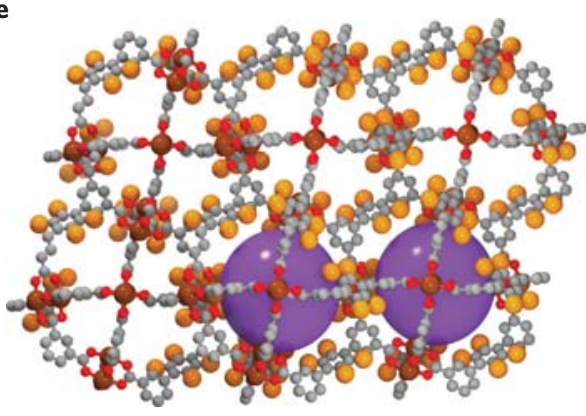

Pseudo-NOTT-107

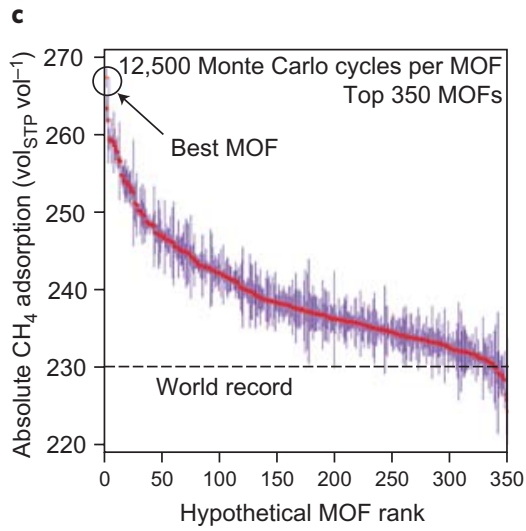

f

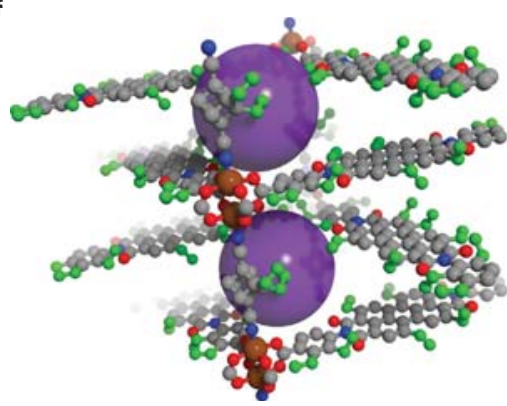

Best MOF

Figure 4 | Adaptive three-stage screening to identify the best MOFs for methane storage. a, In the initial stage, $\sim 137,000$ MOFs were screened for methane storage at 35 bar through short simulations. b,c, The top 5\% of the first stage (b) and then the top 5\% of the second stage (c), highlited in orange, were recalculated using successively longer simulations, which significantly reduced the statistical error each time. In a-c, MOFs are rank-ordered for their methane adsorption abilities (best to worst runs from left to right), with statistical error indicated by purple bars. d,e, Perspective (d) and side (e) views of pseudo-NOTT-107 generated automatically by our method ('pseudo' to distinguish from the empirical structure) and shows methane-storage capacity in the top $2 \%$ of all MOFs generated. f, A hypothetical MOF with very high methane storage at 35 bar; the highest value was 267 vol $_{\text {STP }}$ vol $^{-1}$. In $\mathbf{d}$-f orange and green spheres are the carbon atoms of methyl and ethyl functional groups, respectively, and grey, red, blue and brown spheres represent carbon, oxygen, nitrogen and copper atoms, respectively. The largest pore diameters are indicated by purple spheres, and hydrogen atoms are omitted for clarity. 


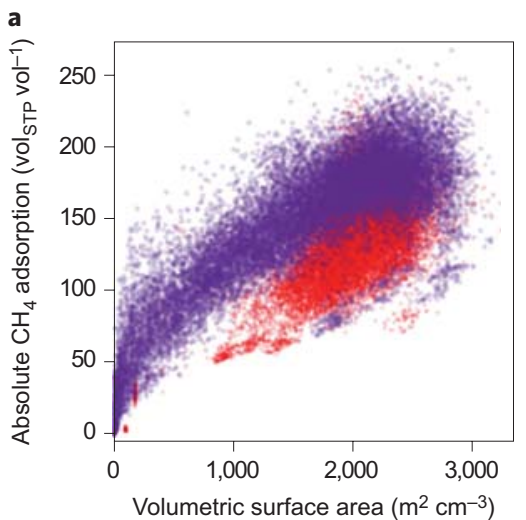

d

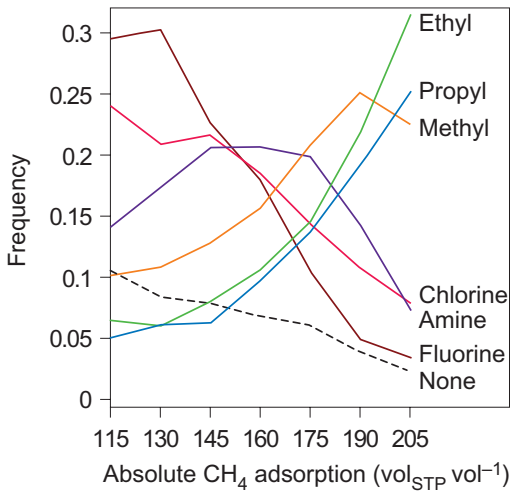

$\stackrel{\text { b }}{\check{I}}$

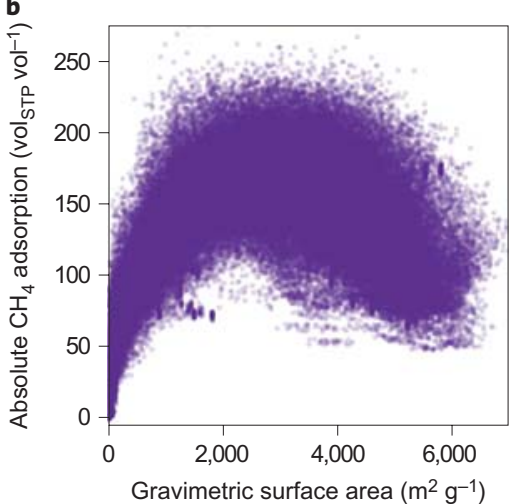

e

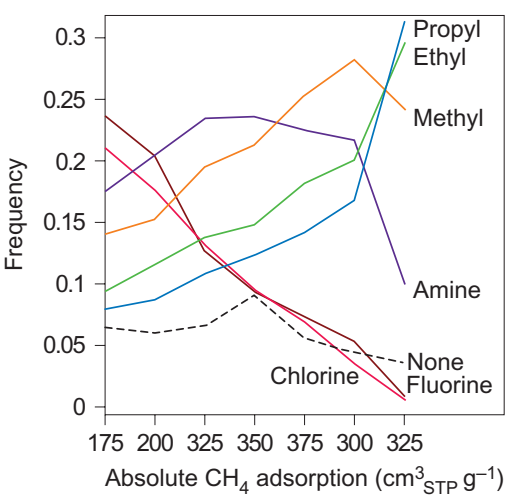

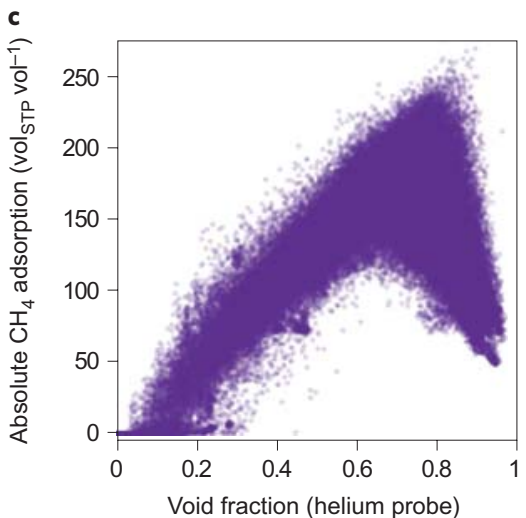

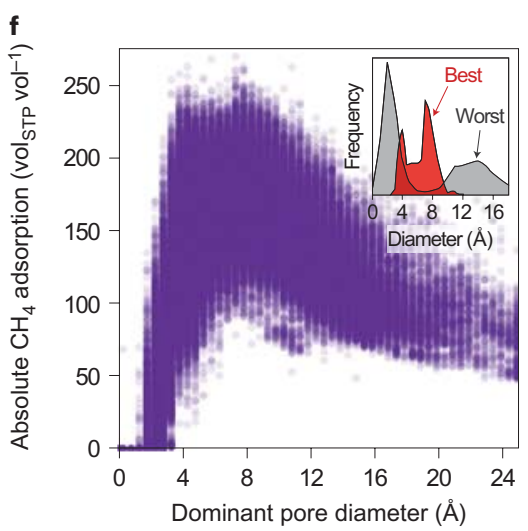

Figure $\mathbf{5}$ | Structure-property relationships obtained from the database of hypothetical MOFs. a, Volumetric methane adsorption shows a clear linear relationship with volumetric surface area. Red dots correspond to MOFs that have enough space to interpenetrate, but are not interpenetrated. $\mathbf{b}$, Methane adsorption initially increases with gravimetric surface area, but then begins to decrease when it has reached the optimal gravimetric surface area of $\sim 2,500-3,000 \mathrm{~m}^{2} \mathrm{~g}^{-1}$. c, A void fraction of $\sim 0.8$ is optimal for volumetric methane uptake at 35 bar. d,e, Methyl, ethyl and propyl groups occur most frequently in MOFs that have volumetric and gravimetric methane adsorption greater than $205 \mathrm{vol}_{\text {STP }} \mathrm{vol}^{-1}$ and $325 \mathrm{~cm}^{3}$ STP $\mathrm{g}^{-1}$, respectively. Halogen functional groups are suboptimal for methane storage; they are weakly represented among the best MOFs for volumetric storage and completely absent from the top MOFs by gravimetric adsorption. $\mathbf{f}$, MOFs in the database have a range of dominant pore sizes. The inset shows that pore sizes of $4 \AA$ and $8 \AA$ are most common in the best MOFs $\left(\mathrm{CH}_{4}\right.$ adsorption $>220 \mathrm{vol}_{\text {STP }}$ vol $\left.^{-1}\right)$, and the worst MOFs $\left(\mathrm{CH}_{4}\right.$ adsorption $<120$ vol STP $\left._{\text {vol }}{ }^{-1}\right)$ specifically exclude pore sizes in the range 4-8 $\AA$.

relation between methane storage and pore size (Fig. 5f) shows that among the best MOFs (storage greater than $220 \mathrm{vol}_{\text {STP }} \mathrm{vol}^{-1}$ ) the most frequent pore sizes are 4 and $8 \AA$, exactly big enough for one or two methane molecules.

Synthesis of high-performance MOFs. Even after computational screening, the procedure can provide a fairly large number of promising predicted structures (for example, $\sim 300$ here). Thus chemical experience is still useful in choosing a smaller subset for which experimental synthesis can be attempted, given constraints of time, cost, available laboratory equipment and reagents, among other criteria. Based on our own constraints, we found a predicted MOF structure in the top $2 \%$ of the database that was a close structural analogue to PCN-14 (Fig. 6a,b), the MOF currently reported to have the highest methane storage at 35 bar (ref. 40). Unbeknown to us at the time of the synthesis, this methylfunctionalized analogue of PCN-14 had been reported previously as the MOF NOTT-107 (ref. 39), but with a significantly different synthesis protocol. Although we found that MOFs with many aromatic rings had a high methane uptake, methyl functional groups had a greater effect. Indeed, GCMC simulations show NOTT-107 (using the pseudo-NOTT-107 structure) to be a slightly better methane-storage material than PCN-14 (simulations using the experimental structure) at $298 \mathrm{~K}$, as shown in Fig. 6c. Absolute storage quantities for NOTT-107 and PCN-14 at 35 bar were calculated to be $213 \mathrm{vol}_{\text {STP }} \mathrm{vol}^{-1}$ and $197 \mathrm{vol}_{\text {STP }} \mathrm{vol}^{-1}$, respectively. Figure 6 displays the excess adsorption (the total amount of gas within the MOF minus the gas that would be present in the same volume without a porous material), which is measured more directly in adsorption experiments.

We observed in experimental measurements at Northwestern University that NOTT-107 had a methane storage capacity $\sim 8 \%$ lower at 35 bar than what had been predicted by our simulations. This could be attributed partly to incomplete pore activation, which is corroborated by a difference between the simulated Brunauer, Emmet and Teller (BET) surface $\operatorname{area}^{51}\left(2,207 \mathrm{~m}^{2} \mathrm{~g}^{-1}\right)$ and the measured BET surface area $\left(1,770 \mathrm{~m}^{2} \mathrm{~g}^{-1}\right)$. The methane adsorption for PCN-14 at $290 \mathrm{~K}$, as measured experimentally by Ma et al. ${ }^{40}$, however, is significantly higher than our model predicts, which is surprising given the similarity of the PCN-14 and NOTT-107 structures (predicted adsorption isotherms of the two MOFs are rather similar, see Fig. $6 \mathrm{c}$ ). Taking the $8 \mathrm{~K}$ lower temperature into account increases the simulated absolute adsorption to $205 \mathrm{vol}_{\text {STP }} \mathrm{vol}^{-1}$ (from 197), but this is still well below the reported value of $230 \mathrm{vol}_{\mathrm{STP}} \mathrm{vol}^{-1}$ (see Supplementary Information for simulation details).

\section{Discussion}

In its current form the method described herein applies only to rigid frameworks. However, as we have demonstrated, the domain of possible rigid frameworks is very large and the comprehensive 
a

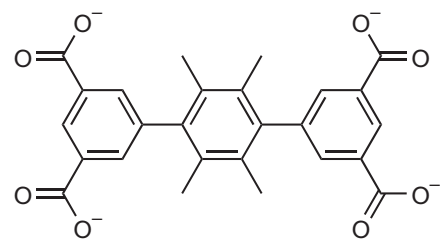

b
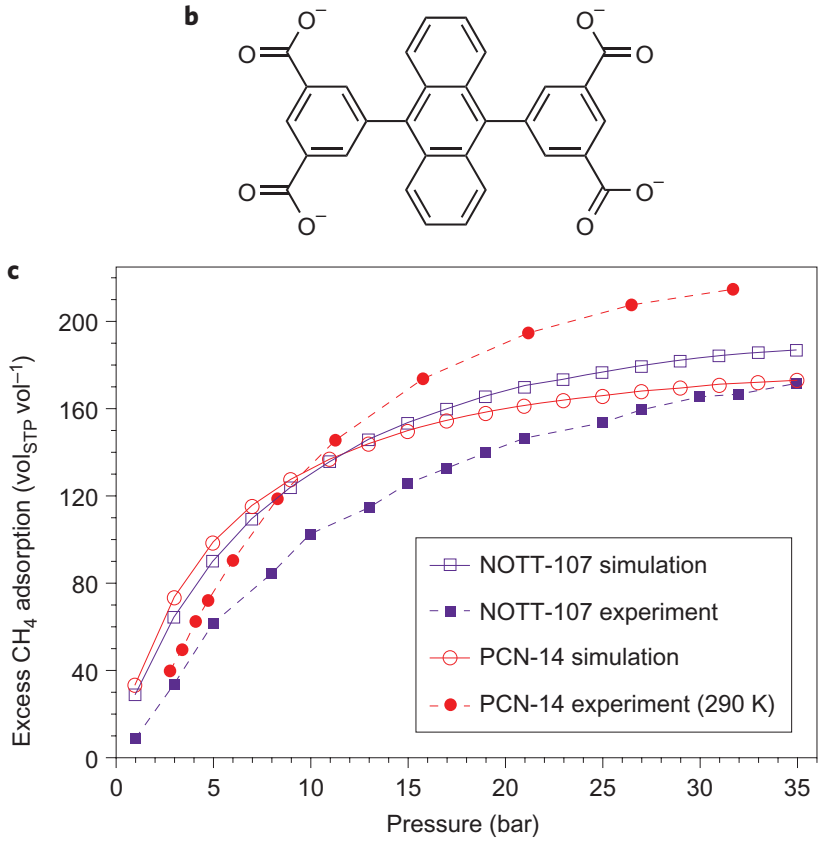

Figure 6 | Comparison of experimental and simulated isotherms for NOTT-107 and PCN 14. a,b, The organic ligand for NOTT-107 (a) is structurally identical to that for PCN-14 (b) except that it bears four methyl groups in the place of two fused aromatic rings. c, Comparison of excess methane adsorption isotherms of NOTT-107 and PCN-14. All isotherm data are at $298 \mathrm{~K}$ except experimental data for PCN-14 taken from ref. 40, which was at $290 \mathrm{~K}$. The simulations used the NOTT-107 structure generated by our method (not optimized) and the PCN-14 structure determined experimentally in ref. 40 .

screening strategy greatly accelerates its exploration. Our method also does not provide, nor guarantee, a synthesis protocol for the generated MOFs. Chemical intuition and synthesis experience are still required to capitalize on the output from the computational generation and screening approach. In the future, it may be possible to extend our method by adding information about the estimated thermodynamics of structure formation to further refine the best candidates. Creating a seamless interface with high-throughput combinatorial experimental synthesis ${ }^{49}$ is also an exciting possibility.

The systematic generation and screening procedure we have developed can be tailored to any application for which computational simulations are able to predict accurately a material property of interest. A probable bottleneck is the time required to compute the property of interest for each structure. This presents a new challenge for areas such as catalysis. Even predicting the adsorption of molecules more complicated than methane may require new tools. For example, Monte Carlo simulations of $\mathrm{CO}_{2}$ adsorption require knowledge of the partial charges on the MOF framework atoms to simulate accurately the adsorption of the quadrupolar $\mathrm{CO}_{2}$. These partial charges are obtained typically from time-consuming quantum mechanical calculations. However, much faster methods for estimating such charges are being developed ${ }^{52,53}$ and will enable the large-scale screening of hypothetical MOFs for carbon capture in the near future.
One can also imagine a more adaptive and creative structuregeneration process. For example, we added propyl building blocks to our input library based on positive results using methyl and ethyl functional groups; similar insights could lead to the design of new building blocks, which could subsequently be fed back into the generator as an iterative optimization strategy.

\section{Conclusion}

When our understanding of materials synthesis approaches the point that we are able to synthesize any material, the new problem becomes which materials should we synthesize? In this report we show how a systematic approach to testing hypothetical MOFs en masse can efficiently focus the attention of synthetic chemists, chemical engineers and materials scientists on the most promising and interesting targets. Although we found several structure-property relationships, undoubtedly we have missed many, and the reader is welcome to search the database online to uncover other correlations. In the future, we envisage utilizing this screening method for applications beyond methane storage, such as carbon capture, hydrogen storage and chemical separations, and subsequently synthesizing improved materials for these processes.

Received 8 June 2011; accepted 3 October 2011; published online 6 November 2011

\section{References}

1. Rosi, N. L. et al. Hydrogen storage in microporous metal-organic frameworks. Science 300, 1127-1129 (2003)

2. Wang, B., Côté, A. P., Furukawa, H., O’Keeffe, M. \& Yaghi, O. M. Colossal cages in zeolitic imidazolate frameworks as selective carbon dioxide reservoirs. Nature 453, 207-211 (2008).

3. Ferey, G. Physical chemistry: trapped gas. Nature 436, 187-188 (2005).

4. Matsuda, R. et al. Highly controlled acetylene accommodation in a metalorganic microporous material. Nature 436, 238-241 (2005).

5. Düren, T., Sarkisov, L., Yaghi, O. M. \& Snurr, R. Q. Design of new materials for methane storage. Langmuir 20, 2683-2689 (2004).

6. Murray, L., Dinca, M. \& Long, J. Hydrogen storage in metal-organic frameworks. Chem. Soc. Rev. 38, 1294-1314 (2009).

7. Seo, J. et al. A homochiral metal-organic porous material for enantioselective separation and catalysis. Nature 404, 982-986 (2000).

8. Bradshaw, D., Prior, T. J., Cussen, E. J., Claridge, J. B. \& Rosseinsky, M. J. Permanent microporosity and enantioselective sorption in a chiral open framework. J. Am. Chem. Soc. 126, 6106-6114 (2004).

9. Düren, T. \& Snurr, R. Q. Assessment of isoreticular metal-organic frameworks for adsorption separations: a molecular simulation study of methane/ $n$-butane mixtures. J. Phys. Chem. B 108, 15703-15708 (2004).

10. Watanabe, T., Keskin, S., Nair, S. \& Sholl, D. S. Computational identification of a metal organic framework for high selectivity membrane-based $\mathrm{CO}_{2} / \mathrm{CH}_{4}$ separations: $\mathrm{Cu}($ hfipbb $)\left(\mathrm{H}_{2} \text { hfipbb }\right)_{0.5}$. Phys. Chem. Chem. Phys. 11, 11389-11394 (2009)

11. Liu, B. et al. Enhanced adsorption selectivity of hydrogen/methane mixtures in metal-organic frameworks with interpenetration: a molecular simulation study. J. Phys. Chem. C 112, 9854-9860 (2008).

12. Li, J-R., Kuppler, R. J. \& Zhou, H-C. Selective gas adsorption and separation in metal-organic frameworks. Chem. Soc. Rev. 38, 1477-1504 (2009).

13. Corma, A. From microporous to mesoporous molecular sieve materials and their use in catalysis. Chem. Rev. 97, 2373-2419 (1997).

14. Lee, J. et al. Metal-organic framework materials as catalysts. Chem. Soc. Rev. 38, 1450-1459 (2009).

15. Lan, A. et al. A luminescent microporous metal-organic framework for the fast and reversible detection of high explosives. Angew. Chem. Int. Ed. 48, 2334-2338 (2009).

16. Franke, M. E. et al. Development and working principle of an ammonia gas sensor based on a refined model for solvate supported proton transport in zeolites. Phys. Chem. Chem. Phys. 5, 5195-5198 (2003).

17. Allendorf, M. D. et al. Stress-induced chemical detection using flexible metal-organic frameworks. J. Am. Chem. Soc. 130, 14404-14405 (2008).

18. Kokotailo, G. T., Lawton, S. L., Olson, D. H. \& Meier, W. M. Structure of synthetic zeolite ZSM-5. Nature 272, 437-438 (1978).

19. Kitagawa, S., Kitaura, R. \& Noro, S-I. Functional porous coordination polymers. Angew. Chem. Int. Ed. 43, 2334-2375 (2004).

20. Li, H., Eddaoudi, M., O’Keeffe, M. \& Yaghi, O. Design and synthesis of an exceptionally stable and highly porous metal-organic framework. Nature 402, 276-279 (1999). 
21. Ferey, G. Hybrid porous solids: past, present, future. Chem. Soc. Rev. 37, 191-214 (2008).

22. Furukawa, H. et al. Ultrahigh porosity in metal-organic frameworks. Science 329, 424-428 (2010)

23. Ferey, G. et al. A chromium terephthalate-based solid with unusually large pore volumes and surface area. Science 309, 2040-2042 (2005).

24. Chae, H. K. et al. A route to high surface area, porosity and inclusion of large molecules in crystals. Nature 427, 523-527 (2004).

25. Farha, O. K. et al. De novo synthesis of a metal-organic framework material featuring ultrahigh surface area and gas storage capacities. Nature Chem. 2, 944-948 (2010).

26. Zaworotko, M. J. Materials science: designer pores made easy. Nature 451, 410-411 (2008).

27. Moulton, B. \& Zaworotko, M. J. From molecules to crystal engineering: supramolecular isomerism and polymorphism in network solids. Chem. Rev. 101, 1629-1658 (2001)

28. Ockwig, N. W., Delgado-Friedrichs, O., O’Keeffe, M. \& Yaghi, O. M. Reticular chemistry: occurrence and taxonomy of nets and grammar for the design of frameworks. Acc. Chem. Res. 38, 176-182 (2005).

29. Yaghi, O. M. et al. Reticular synthesis and the design of new materials. Nature 423, 705-714 (2003).

30. Eddaoudi, M. et al. Systematic design of pore size and functionality in isoreticular MOFs and their application in methane storage. Science 295, 469-472 (2002).

31. Eddaoudi, M. et al. Modular chemistry: secondary building units as a basis for the design of highly porous and robust metal-organic carboxylate frameworks. Acc. Chem. Res. 34, 319-330 (2001).

32. Perry, J. J. IV \& Perman, J. A. Design and synthesis of metal-organic frameworks using metal-organic polyhedra as supermolecular building blocks. Chem. Soc. Rev. 38, 1400-1417 (2009).

33. Vaidhyanathan, R. et al. Direct observation and quantification of $\mathrm{CO}_{2}$ binding within an amine-functionalized nanoporous solid. Science 330, 650-653 (2010).

34. Frost, H., Düren, T. \& Snurr, R. Q. Effects of surface area, free volume, and heat of adsorption on hydrogen uptake in metal-organic frameworks. J. Phys. Chem. B 110, 9565-9570 (2006).

35. Earl, D. J. \& Deem, M. W. Toward a database of hypothetical zeolite structures. Ind. Eng. Chem. Res. 45, 5449-5454 (2006).

36. Haldoupis, E., Nair, S. \& Sholl, D. S. Pore size analysis of $>250,000$ hypothetical zeolites. Phys. Chem. Chem. Phys. 13, 5053-5060 (2011).

37. Baerlocher, Ch. \& McCusker, L. B. Database of zeolite structures: http://www.iza-structure.org/databases/.

38. Haldoupis, E., Nair, S. \& Sholl, D. S. Efficient calculation of diffusion limitations in metal organic framework materials: a tool for identifying materials for kinetic separations. J. Am. Chem. Soc. 7258-7539 (2010).

39. Lin, X. et al. High capacity hydrogen adsorption in $\mathrm{Cu}(\mathrm{II})$ tetracarboxylate framework materials: the role of pore size, ligand functionalization, and exposed metal sites. J. Am. Chem. Soc. 131, 2159-2171 (2009).

40. Ma, S. et al. Metal-organic framework from an anthracene derivative containing nanoscopic cages exhibiting high methane uptake. J. Am. Chem. Soc. 130, 1012-1016 (2008).

41. Chui, S. S-Y., Lo, S. M-F., Charman, J. P. H., Orpen, A. G. \& Williams, I. D. A chemically functionalizable nanoporous material $\left[\mathrm{Cu}_{3}(\mathrm{TMA})_{2}\left(\mathrm{H}_{2} \mathrm{O}\right)_{3}\right]_{n}$. Science 283, 1148-1150 (1999).

42. Barthelet, K., Marrot, J., Riou, D. \& Férey, G. A breathing hybrid organicinorganic solid with very large pores and high magnetic characteristics. Angew. Chem. Int. Ed. 41, 281-284 (2002).
43. Rappé, A. K., Casewit, C. J., Colwell, K. S., Goddard, W. A. III \& Skiff, W. M. UFF, a full periodic table force field for molecular mechanics and molecular dynamics simulations. J. Am. Chem. Soc. 114, 10024-10035 (1992).

44. Materials Studio v 5.0. Accelrys Software Inc., San Diego, California 92121, USA.

45. Düren, T., Bae, Y.-S. \& Snurr, R. Q. Using molecular simulation to characterise metal-organic frameworks for adsorption applications. Chem. Soc. Rev. $\mathbf{3 8}$, 1237-1247 (2009).

46. Menon, V. C. \& Komarneni, S. Porous adsorbents for vehicular natural gas storage: a review. J. Porous Mater. 5, 43-58 (1998).

47. Zhou, W. Methane storage in porous metal-organic frameworks: current records and future perspectives. Chem. Rec. 10, 200-204 (2010).

48. Senkovska, I. \& Kaskel, S. High pressure methane adsorption in the metal-organic frameworks $\mathrm{Cu}_{3}(\mathrm{btc})_{2}, \mathrm{Zn}_{2}(\mathrm{bdc})_{2}$ dabco, and $\mathrm{Cr}_{3} \mathrm{~F}\left(\mathrm{H}_{2} \mathrm{O}\right)_{2} \mathrm{O}(\mathrm{bdc})_{3}$. Micropor. Mesopor. Mat. 112, 108-115 (2008).

49. Deng, H. et al. Multiple functional groups of varying ratios in metal-organic frameworks. Science 327, 846-850 (2010).

50 . Wu, H. et al. Metal-organic frameworks with exceptionally high methane uptake: where and how is methane stored? Chem. Eur. J. 16, 5205-5214 (2010).

51. Walton, K. S. \& Snurr, R. Q. Applicability of the BET method for determining surface areas of microporous metal-organic frameworks. J. Am. Chem. Soc. 129, 8552-8556 (2007).

52. Xu, Q. \& Zhong, C. A general approach for estimating framework charges in metal-organic frameworks. J. Phys. Chem. C 114, 5035-5042 (2010).

53. Wilmer, C. E. \& Snurr, R. Q. Towards rapid computational screening of metal-organic frameworks for carbon dioxide capture: calculation of framework charges via charge equilibration. Chem. Eng. J. 171, 775-781 (2011).

\section{Acknowledgements}

The authors thank Y. Aktan and M. Tsao for the supporting calculations, as well as M.A. Wilmer for significant contributions to the website interface to the hypothetical MOF database. R.Q.S. acknowledges support by the Defense Threat Reduction Agency (grant HDTRA1-09-1-0007). Computational work was supported through the resources provided by Information Technology at Northwestern University as part of its shared cluster program, Quest. J.T.H. and O.K.F. acknowledge support from the US Deptartment of Energy, Office of Science, Basic Energy Sciences program (grant DE-FG02-08ER15967) and the Northwestern Nanoscale Science and Engineering Center. C.E.W. acknowledges support from a Fellowship from the Initiative for Sustainability and Energy at Northwestern and a Ryan Fellowship from the Northwestern University International Institute for Nanotechnology.

\section{Author contributions}

C.E.W. designed the research, implemented the algorithms and performed the simulations with assistance from M.L. and guidance from R.Q.S. O.K.F helped in ligand and node selection. The NOTT-107 ligand was synthesized by O.K.F. and C.Y.L. NOTT-107 was synthesized and activated by O.K.F. NOTT-107 physical characterization was done by B.G.H. Experimental data interpretation and MOF-activation methodology was developed by J.T.H. and O.K.F. All authors discussed the results, contributed to writing the manuscript and commented on it.

\section{Additional information}

The authors declare no competing financial interests. Supplementary information accompanies this paper at www.nature.com/naturechemistry. Reprints and permission information is available online at http://www.nature.com/reprints. Correspondence and requests for materials should be addressed to R.Q.S 